\title{
Nitric oxide-sphingolipid interplays in plant signaling: a new enigma from the Sphinx?
}

\author{
Isabelle Guillas ${ }^{1,2}$, Juliette Puyaubert ${ }^{1,2}$ and Emmanuel Baudouin ${ }^{1,2}$ * \\ 1 UR 5, Laboratoire de Physiologie Cellulaire et Moléculaire des Plantes, Université Pierre et Marie Curie - Paris 6, Paris, France \\ ${ }^{2}$ EAC 7180, Laboratoire de Physiologie Cellulaire et Moléculaire des Plantes, Centre National de la Recherche Scientifique, Paris, France
}

\author{
Edited by: \\ John Hancock, University of the West \\ of England, UK \\ Reviewed by: \\ John Hancock, University of the West \\ of England, UK \\ Patrice Thuleau, Centre National de la \\ Recherche Scientifique, France

\section{*Correspondence:} \\ Emmanuel Baudouin, UR 5, \\ Laboratoire de Physiologie Cellulaire \\ et Moléculaire des Plantes, Université \\ Pierre et Marie Curie - Paris 6, \\ Bâtiment C/3 Boîte courrier 156, 4 \\ place Jussieu, F-75252 Paris Cédex \\ 05, France; EAC 7180, Laboratoire de \\ Physiologie Cellulaire et Moléculaire \\ des Plantes, Centre National de la \\ Recherche Scientifique, Bâtiment C/3 \\ Boîte courrier 156, 4 place Jussieu \\ F-75252 Paris Cédex 05, France \\ e-mail:emmanuel.baudouin@upmc.fr
}

Nitric oxide (NO) emerged as one of the major signaling molecules operating during plant development and plant responses to its environment. Beyond the identification of the direct molecular targets of NO, a series of studies considered its interplay with other actors of signal transduction and the integration of NO into complex signaling networks. Beside the close relationships between $\mathrm{NO}$ and calcium or phosphatidic acid signaling pathways that are now well-established, recent reports paved the way for interplays between NO and sphingolipids (SLs). This mini-review summarizes our current knowledge of the influence $\mathrm{NO}$ and SLs might exert on each other in plant physiology. Based on comparisons with examples from the animal field, it further indicates that, although SL-NO interplays are common features in signaling networks of eukaryotic cells, the underlying mechanisms and molecular targets significantly differ.

Keywords: sphingolipids, ceramides, long chain bases, nitric oxide, plant, signaling, abiotic and biotic stresses

\section{INTRODUCTION}

Nitric oxide (NO) is a pleiotropic actor of signaling cascades in eukaryotes (Baudouin, 2011; Martínez-Ruiz et al., 2011). The last 15 years have provided a plethora of examples for the involvement of NO essentially at all stages of plant development or in response to most environmental cues (Baudouin, 2011; Mur et al., 2013). De facto cardinal questions such as the origin, mode of action, or integration of NO signal into regulatory networks became of broad interest for plant biologists (Besson-Bard et al., 2008; Mur et al., 2013). The complex chemistry of NO enables its reactivity toward an array of biological molecules including proteins, DNA, and lipids (Calcerrada et al., 2011). In particular specific protein targets that undergo NO-based post-translational modifications (PTM; such as S-nitrosylation and/or nitration, that implicate cysteine and tyrosine residues, respectively) are crucial to convert NO signal into proper physiological responses (Jacques et al., 2013; Kovacs and Lindermayr, 2013). This aspect of NO signaling has been paid much attention in plants in the recent years and led to the identification of hundreds of proteins undergoing such NObased PTM, and, in a few cases, to a further characterization of the targeted proteins (Kovacs and Lindermayr, 2013). Beyond this direct modus operandi, increasing evidence shed light on the intricate relationships between $\mathrm{NO}$ and other intracellular signals such as $\mathrm{Ca}^{2+}$, cGMP, phosphatidic acid (PtdOH), or reactive oxygen species (ROS), that trigger, mediate and/or modulate NO signal in response to specific stimuli (Gaupels et al., 2011). A recent review addressed this concern with the example of $\mathrm{NO} / \mathrm{Ca}^{2+}$ interactions and illustrated some molecular mechanisms through which NO and $\mathrm{Ca}^{2+}$ signaling could regulate each other (Jeandroz et al., 2013). Although the underlying mechanisms are less documented, interplays have also been evidenced between $\mathrm{NO}$ and the lipid signal PtdOH (Laxalt et al., 2007; Distéfano et al., 2012).

Recently, sphingolipids (SLs), another class of well-known signaling lipids in mammal cells, were ascribed important signaling functions in plants (Berkey and Xiao, 2012; Markham et al., 2013). Numerous examples evidenced crosstalks between SL and NO during (patho)physiological processes in animals (Perrotta et al., 2008). Seminal reports suggest that some interactions could also operate in plants. This mini-review presents our current knowledge of the interactions existing between NO and SL signaling in plants, and put it in perspective with well-documented examples from the animal field.

\section{SPHINGOLIPID SYNTHESIS AND SIGNALING IN PLANTS}

The generic term SLs designate both membrane-located complex SL (glycosylceramides, inositol-phosphoceramides, and glycosylinositol-phosphorylceramides in plants) and their metabolic precursors, i.e., long chain bases (LCB) and ceramides (Cer; Pata et al., 2010). They therefore constitute a diverse family of hundreds of molecular entities (Cacas etal., 2012). Adding to this complexity, a subset of LCB and Cer can get phosphorylated by specific LCB and Cer kinases, respectively. Finally the relative amount of the different SL species is not steady, but might undergo fluctuations due to the regulation of SL synthesis, degradation, 
and/or phosphorylation/dephosphorylation leading to an overrepresentation of specific SL (Kihara et al., 2007). Therefore, far away from the early picture of SL being static constitutive entities, the sphingolipidome now emerges as dynamic, possibly modified in response to inside and outside signals and thereafter prompting a range of physiological responses (Markham et al., 2013).

Parallel to the decoding of sphingolipidome, studies conducted during the last decade brought tangible evidences for SL function in signaling networks operating during plant development and responses to environmental cues (Berkey and Xiao, 2012; Markham et al., 2013). Best documented are signaling functions for the precursors of complex SL, i.e., LCB and Cer. For instance LCB and Cer participate in the induction and/or control of plant cell death as illustrated by several studies in which LCB/Cer content was modified by exogenous treatments or the disruption of key genes of SL metabolism (Liang et al., 2003; Lachaud et al., 2010; Saucedo-Garcia et al., 2011; Ternes et al., 2011). The biological relevance of LCB/Cer-triggered cell death has been assumed for plant-pathogen interactions as (i) transient increases of LCB content are observed upon pathogen infection and (ii) pathogen-induced cell death is altered in mutants of SL metabolism (Brodersen et al., 2002; Liang et al., 2003; Peer et al., 2010). Noteworthy complex membrane-located SL also participate in pathogen-triggered cell death (Wang et al., 2008). Furthermore, whereas LCB/Cer promote cell death, phosphorylated LCB (LCB-P) and Cer (Cer-P) prevent cell death (Liang et al., 2003; Shi et al., 2007; Alden et al., 2011). As in mammal cells, the tight control of LCB/LCB-P and Cer/Cer-P equilibrium, and more generally of SL metabolism, is therefore a crucial aspect of plant cell homeostasis keeping it alive or bringing it to death. Whereas the function of Cer/Cer-P has only been investigated in relation with cell death, the role of LCB/LCB-P likely exceeds this limited context. Indeed, mutants of LCB/LCB-P metabolism present altered responses to abiotic stresses unrelated to cell viability. For instance LCB-P have been implicated in a abscisic acid (ABA)-dependent pathway regulating stomatal aperture and drought stress tolerance (Ng et al., 2001; Coursol et al., 2003; Worrall et al., 2008). LCB-P have also been implicated in cold, salt, and oxidative stress responses (Dutilleul et al., 2012; Zhang et al., 2012). These studies have identified several upstream and downstream elements of the SL signaling cascade including $\mathrm{Ca}^{2+}$, heterotrimeric $\mathrm{G}$ proteins, ROS, and the MAP kinase AtMPK6. Recent data also suggest that $\mathrm{PtdOH}$ signaling can act in a coordinated way with SL (Guo et al., 2011, 2012). Whether these signals are ubiquitous elements of SL signaling is currently unknown.

Less documented in plants are the signaling functions of SL related to their particular location within membrane microdomains (rafts). Rafts are not only enriched in SL and sterols, but also present a particular protein composition (SimonPlas et al., 2011; Cacas et al., 2012). Indeed, plant membrane rafts are rich in signaling-related proteins (Morel et al., 2006; Lefebvre et al., 2007). Such signaling proteins might not be permanent raft residents but rather temporarily recruited following stimulus perception (Minami et al., 2009; Li et al., 2011). Therefore, rafts emerged as potent signaling platforms and the dynamic modification of membrane structure/composition is probably involved in signal transduction during plant development and response to environmental cues. For instance alterations of membrane integrity via defects in SL composition led to strong developmental phenotypes due to auxin carrier mislocalization (Roudier et al., 2010; Markham et al., 2011). Moreover analysis of the SL and raft abundance and the raft lipid/protein composition during plant acclimation to cold evidenced a close correlation between SL and raft dynamics (Minami et al., 2010). Although the mechanisms underlying the remodeling of rafts is far from being solved, SL have been demonstrated as key components for raft formation in animal membranes (Filippov et al., 2006). As proposed by Cacas et al. (2012), this function of membrane SL is likely conserved in plants, therefore outlining a possible link between SL-based regulation of raft formation and/or structure and SL-triggered signaling events.

\section{INTERPLAYS BETWEEN SL AND NO SIGNALING: SOME LESSONS FROM MAMMAL CELLS}

Studies in the animal field initiated in the late 1990s brought to light interconnections between SL and NO signaling in (patho-) physiological situations (reviewed in Huwiler and Pfeilschifter, 2003; Igarashi and Michel, 2008; Perrotta and Clementi, 2010). The models hypothesized from these studies principally implicate Cer and sphingosine 1-P (S1P), the major LCB signal in animal cells (Figures 1A,B). First, NO can regulate sphingomyelinases (SMase) that generate the formation of Cer from sphingomyelin (SM), a major membrane SL in mammal cells (Figure 1A; Perrotta et al., 2008). Interestingly NO might regulate SMase activities in a different way, depending on the intracellular NO level. On the one hand low physiological concentrations of NO lead to the inhibition of SMases, thereby preventing cell death in a large range of (patho)physiological models by reducing the intracellular Cer concentration (Falcone et al., 2004; Perrotta et al., 2007). On the other hand high levels of NO lead to the increase of Cer concentration, thereby driving cells to apoptosis (Takeda et al., 1999; Pilane and LaBelle, 2004). In this last case (Figure 1A), NO promotes (i) the activation of SMases that generate Cer and (ii) represses Cer degradation via the inhibition of ceramidase activities (Huwiler et al., 1999; Franzen et al., 2002). How NO up-regulates SMases and down-regulates ceramidases under such conditions is currently unknown. A SMase isoform has been recently identified as $S$-nitrosylated in mouse, thus providing a possible mechanism for SMase regulation by high NO concentration (Kohr et al., 2011). Under low NO concentrations SMase inhibition is likely indirect and involves a cGMP/PKG-dependent pathway, possibly in relation with the regulation of SMase intracellular localization (Falcone et al., 2004; Perrotta and Clementi, 2010).

A second model is illustrated by the regulation of NO formation by the endothelial NO synthase (eNOS) mediated by S1P (Figure 1B; Igarashi and Michel, 2008). At least two mechanisms are at play in this process. Firstly the association of eNOS with and its inhibition by caveolin, a transmembrane protein located in the raft-related caveolae microdomains, is reverted by a $\mathrm{Ca}^{2+}$ dependent mechanism mediated by S1P (Igarashi and Michel, 2008). Secondly S1P via its binding to S1P receptors activates a signaling cascade involving AMP-activated protein kinase, Rac1 $\mathrm{G}$ protein, $\mathrm{PI}_{3}$ kinase, and Akt kinase, ending up at eNOS phosphorylation and activation (Levine et al., 2007). Strikingly most 


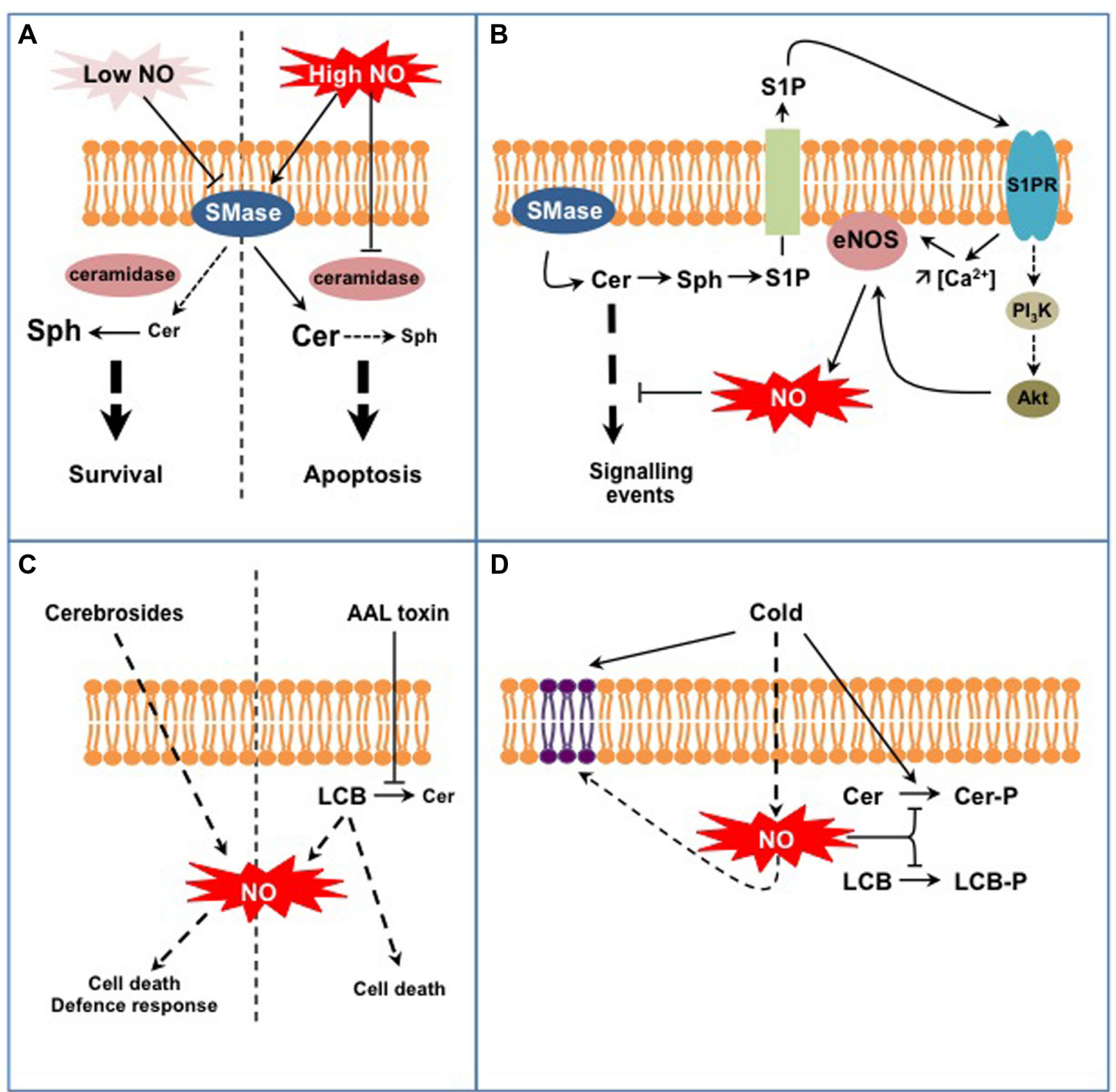

FIGURE 1 | Examples of interplays between SL and NO signaling in animal (A,B) and plant cells (C,D). (A) NO regulates Cer formation from sphingomyelin in a dose-dependent process. Low NO concentrations inhibit sphingomyelinase activity (SMase) leading to low Cer levels that are further degraded to sphingosine (Sph) by ceramidases. High NO concentrations stimulate SMase activities while inhibiting ceramidases, therefore leading to high Cer levels. This differential control of SMases by NO participates in Cer-dependent cell survival or death. (B) Cer formation indirectly triggers endothelial NOS (eNOS) activation and NO formation. Sphingosine-1P (S1P) is formed from Cer degradation and subsequent phosphorylation of Sph. S1P is externalized and perceived on the outer cell surface by specific
S1P receptors (S1PR). Activated S1PR trigger eNOS activation via an increase of cytosolic $\mathrm{Ca}^{2+}$ concentration and/or via the regulation of the $\mathrm{Pl}_{3} \mathrm{~K} /$ Akt signaling pathway. eNOS-evoked NO eventually down-regulates Cer-activated signaling pathways. (C) Complex SL (fungal cerebrosides), SL precursors (LCB), or SL analogs (AAL fungal toxin) act as potent inducers of $\mathrm{NO}$ formation. Such NO production might participate in specific aspects of SL-triggered cell death or defense responses. (D) Plant exposure to cold triggers the formation of NO that down-regulates the synthesis of phospho-SL (i.e., Cer-P and LCB-P). In addition, NO participates in the modification of membrane SL content resulting from low temperature exposure. of these proteins are located within caveolae, in close vicinity with eNOS. Such common location also accounts when considering the origin of S1P. As exemplified in TNF $\alpha$-stimulated HeLa cells, S1P originates from Cer released from SM by a specific SMase isoform (Barsacchi et al., 2003). Cer are subsequently deacylated by ceramidases into sphingosine that gets phosphorylated to S1P. Noteworthy the SMase isoform involved in this model is also located in caveolae together with eNOS (Perrotta and Clementi, 2010). Ultimately eNOS-evoked NO counteracts the apoptotic effects of Cer by inhibiting Cer signaling pathway. 
These examples point out the intricate network involving NO and SL in mammal cells. Part of this complexity resides in the diverse isoforms of SMases and ceramidases that undergo different NO-based regulations. Thereby, NO might contribute as an enhancer or a down-regulator of Cer signaling. These examples finally underline that the interplay between NO and SL signaling is not unidirectional, but can also involve bi-directional signaling according to the cellular response examined.

\section{INTERPLAYS BETWEEN SL AND NO SIGNALING IN PLANTS: PROMISES FROM DAWN}

At first glance, the models depicted above seem not transposable to plants as most of the molecular actors mentioned are absent from plant cells (e.g., SM, SMases, S1P receptors, eNOS). Nevertheless, several lines of evidence indicate the existence of similar interplays between SL and NO signaling in plants. First, studies have reported the capacity of SL-related molecules to trigger $\mathrm{NO}$ synthesis (Figure 1C). Wang et al. (2007, 2009) evidenced that treatments with cerebrosides from the fungal pathogen Fusarium sp IFB-121 induce NO formation in Taxus yunnanensis and Artemisia annua. Cerebrosides are complex membrane SL widely found in soilborne fungi and are considered as pathogenassociated molecular patterns (PAMP; Umemura et al., 2004). In this context, cerebroside-evoked NO triggers the synthesis of secondary metabolites, i.e., taxol and artemisin (Wang et al., 2007, 2009). In addition to SL-related elicitors, some pathogenic fungi produce toxins structurally analogous to LCB, such as AAL toxin from Alternaria alternata f.sp. lycopersici or fumonisin B1 (FB1) from Fusarium moniliforme. Although the formation of $\mathrm{NO}$ in response to these toxins has not been directly evidenced, AALtriggered cell death was blocked by an inhibitor of mammalian NOS suggesting that NO was required for AAL response (Gechev et al., 2004). Being LCB analogs, AAL and FB1 toxins block Cer synthesis and provoke free LCB accumulation (Abbas et al., 1994). Interestingly, Da Silva et al. (2011) recently showed that exogenous treatments with LCB triggered NO formation in tobacco cells. Nevertheless the biological outcome of LCB-stimulated NO production remains obscure as $\mathrm{NO}$ was not required for LCB-induced cell death. Although seminal, these studies require further investigations to establish the biological relevance of SL-triggered NO formation. For instance, one has to establish if specific SL structural features are required to trigger $\mathrm{NO}$ production, as reported for $\mathrm{H}_{2} \mathrm{O}_{2}$ synthesis (Shi et al., 2007). As plants lack bona fide NOS, the source of SL-evoked NO should be hunted, together with the mechanisms underlying its activation by SL. Finally it is noteworthy that the data available rely on exogenous treatment of plant material with SL-related molecules. One has therefore to examine if and how endogenous modifications of SL homeostasis might induce NO production.

Conversely to the regulation of NO formation by SL, recent data indicate that NO regulates specific aspects of SL metabolism in plants (Figure 1D). In particular it may participate in the fine-tuning of the equilibrium between LCB/Cer and LCB-P/CerP. This was evidenced for Arabidopsis response to cold where two phosphorylated SL species (i.e., the LCB phytosphingosinephosphate and a putative Cer-P) are rapidly and transiently formed (Cantrel et al., 2011; Dutilleul et al., 2012). In this context cold-evoked NO functions as a negative regulator of phospho-SL formation (Cantrel et al., 2011). How NO regulates phospho-SL formation during cold-stress response remains unclear. Firstly NO could impact the activity of kinases or phosphatases metabolizing LCB-P and Cer-P. For instance sphingosine kinase 1 (SPHK1) is regulated by NO in human endothelial cells (Schwalm et al., 2010). So far only S1P lyase, which catalyses the degradation of LCB-P, has been identified as a target for NO-based PTM and the biological significance of this modification remains to be established (Zhan and Desiderio, 2009). The regulation of LCB-metabolizing enzymes has been poorly studied in plants and further investigations are therefore required to decipher if $\mathrm{NO}$ can directly regulate these enzymes. Secondly NO might modify the availability of LCB/Cer kinase substrates. Supporting this possibility, Guillas et al. (2013) evidenced that an Arabidopsis mutant line overexpressing a non-symbiotic hemoglobin, and thereby exhibiting low NO levels, over-accumulates phytosphingosine. The levels of phytosphingosine were further increased after cold exposure and might afford for the highest rate of phytosphingosine-P formation observed in this mutant (Cantrel et al., 2011). Interestingly, the analysis revealed another facet of the SL response affected in this mutant. Indeed, whereas the overall amount of LCB was strongly lowered by cold exposure in WT plants, it was drastically increased in the mutant line (Guillas et al., 2013). These data therefore suggest that NO might participate in the regulation of more complex sphingolipidome modifications associated with cold response.

As for SL-triggered NO formation, the example presented above questions about the ubiquity of SL-NO interplay in diverse physiological contexts and the underlying mechanisms at work. Although direct connections have not been established yet, it is likely that SL-NO crosstalks participate in ABA signaling (Zhang et al., 2009; Guo and Wang, 2012). In this framework PtdOH metabolism and signaling could be crucial to interlink SL and NO signaling. Indeed ABA activates phospholipase $\mathrm{D} \alpha 1$ (PLD $\alpha 1)$ to synthesize $\mathrm{PtdOH}$ and thereby triggers NO formation (Zhang et al., 2009; Uraji et al., 2012). Strikingly PLD $\alpha 1$ is also a target for LCB-P that stimulate PtdOH synthesis (Guo and Wang, 2012). This apparent simplicity turns to complexity when considering that (i) PtdOH generated by $P L D \alpha 1$ interacts with and further stimulates the LCB kinase SPHK1 (Guo et al., 2012) and (ii) that a ABA-triggered NO production is also required for the activation of Phospholipase $\mathrm{D} \delta$ and PtdOH synthesis (Distéfano et al., 2012). In this intricate signaling network, further investigations should now examine the consequences of alterations of NO or SL signaling on each other to clearly establish possible direct NO-SL crosstalks. The interaction between Arabidopsis and the phytopathogenic bacteria Pseudomonas syringae is another context where NO-SL interactions are likely. On the one hand studies carried out on this pathosystem led to the pioneering demonstration of NO as a signal in plants (Delledonne et al., 1998). On the other hand it provided the first example of dynamic changes of LCB level triggered by biotic stress in plants (Peer et al., 2010). Although the interplay of $\mathrm{NO}$ and LCB has not been addressed yet in this system, it opens the possibility that NO regulates LCB metabolism as suggested above for cold stress, and/or that LCB trigger NO production as reported for ROS (Peer et al., 2011). Besides interacting within signaling 
networks or interfering with each other metabolism, NO and SL might interfere at the level of protein trafficking toward membranes. In the case of auxin bioactivity, defects in either SL or NO metabolism lead to misaddressing or degradation of the auxin efflux transporter PIN1 and thereby to altered development of root system (Fernández-Marcos et al., 2011; Markham et al., 2011; Yang etal., 2013). Due to the recent involvement of NO in vesicle trafficking in roots (Lombardo and Lamattina, 2012), further analysis of its interplay with SL in this context might shed light on unexplored roles for $\mathrm{NO}$ in plant cell biology.

\section{CONCLUSION}

Increasing evidence plead for functional interplays between NO and lipid signaling and indirectly bring to forestage the role of biological membranes in NO biology. As exemplified in mammals and plants, SL signals generated by the catabolism or as intermediates of the synthesis of complex membrane SL, constitute new elements of the NO signaling network in a variety of

\section{REFERENCES}

Abbas, H. K., Tanaka, T., Duke, S. O., Porter, J. K., Wray, E. M., Hodges, L., et al. (1994). Fumonisinand AAL-toxin-induced disruption of sphingolipid metabolism with accumulation of free sphingoid bases. Plant Physiol. 106, 1085-1093.

Alden, K. P., Dhondt-Cordelier, S., McDonald, K. L., Reape, T. J., Ng, C. K.-Y., McCabe, P. F., et al. (2011). Sphingolipid long chain base phosphates can regulate apoptotic-like programmed cell death in plants. Biochem. Biophys. Res. Commun. 410, 574-580. doi: 10.1016/j.bbrc.2011.06.028

Barsacchi, R., Perrotta, C., Bulotta, S., Moncada, S., Borgese, N., and Clementi, E. (2003). Activation of endothelial nitric-oxide synthase by tumor necrosis factor-alpha: a novel pathway involving sequential activation of neutral sphingomyelinase, phosphatidylinositol-3' kinase, and Akt. Mol. Pharmacol. 63, 886-895. doi: $10.1124 / \mathrm{mol} .63 .4 .886$

Baudouin, E. (2011). The language of nitric oxide signalling. Plant Biol. 13, 233-242. doi: 10.1111/j.14388677.2010.00403.x

Berkey, R., and Xiao, S. (2012). Sphingolipids and plant defense/disease: the "death" connection and beyond. Front. Plant Sci. 3:68. doi: 10.3389/fpls.2012.00068

Besson-Bard, A., Pugin, A., and Wendehenne, D. (2008). New insights into nitric oxide signaling in plants. Annu. Rev. Plant Biol. 59, 21-39. doi: 10.1146/annurev.arplant.59.032607. 092830

Brodersen, P., Petersen, M., Pike, H. M., Olszak, B., Skov, S., Odum, N., et al. (2002). Knockout of Arabidopsis accelerated-cell-death11 encoding a sphingosine transfer protein causes activation of programmed cell death and defense. Genes Dev. 16, 490-502. doi: 10.1101/gad.218202

Cacas, J.-L., Furt, F., Le Guédard, M., Schmitter, J.-M., Buré, C., Gerbeau-Pissot, P., etal. (2012). Prog. Lipid Res. 51, 272-299. doi: 10.1016/j.plipres.2012.04.001

Calcerrada, P., Peluffo, G., and Radi, R. (2011). Nitric oxide-derived oxidants with a focus on peroxynitrite: molecular targets, cellular responses, and therapeutic implications. Curr. Pharm. Des. 17, 3905-3932. doi: 10.2174/138161211798357719

Cantrel, C., Vazquez, T., Puyaubert, J., Reze, N., Lesch, M., Kaiser, W. M., etal. (2011). Nitric oxide participates in cold-responsive phosphosphingolipid formation and gene expression in Arabidopsis thaliana. New Phytol. 189, 415-427. doi: 10.1111/j.1469-8137.2010.03500.x

Coursol, S., Fan, L. M., Le Stunff, H., Spiegel, S., Gilroy, S., and Assmann, S. M. (2003). Sphingolipid signalling in Arabidopsis guard cells involves heterotrimeric G proteins. Nature 423, 651-654. doi: 10.1038/nature0 1643

Da Silva, D., Lachaud, C., Cotelle, V., Brière, C., Grat, S., Mazars, C., et al. (2011). Nitric oxide production is not required for dihydrosphingosineinduced cell death in tobacco BY-2 cells. Plant Signal. Behav. 6, 736-739. doi: 10.4161/psb.6.5.15126

Delledonne, M., Xia, Y., Dixon, R. A., and Lamb, C. (1998). Nitric oxide functions as a signal in plant disease resistance. Nature 394, 585-588. doi: $10.1038 / 29087$ Lipids of plant membrane rafts.

physiological processes. The rising interest for SL and NO signaling in plants will undoubtedly provide soon new examples of this interplay. Future investigations should help unravel the mechanisms underlying such NO-SL signaling crosstalks. In particular a direct regulation of enzymes of the NO and SL pathway by SL and NO, respectively should be evaluated. As observed in mammals, this might include modulation of activity but also regulation of protein targeting. Finally it is likely that NO, which is liposoluble, does not only interplay with SL signaling within the cytosol, but also within the biological membranes. As it might deeply affect the activity and/or targeting of membrane-located proteins and the overall membrane structure, attention should now be paid to NO signaling within the lipid phase.

\section{ACKNOWLEDGMENTS}

This work was supported by the Agence Nationale de la Recherche (BLAN07-2_184783), the Centre National de la Recherche Scientifique, and the Université Pierre et Marie Curie - Paris 6.

Distéfano, A. M., Scuffi, D., GarcíaMata, C., Lamattina, L., and Laxalt, A. M. (2012). Phospholipase D $\delta$ is involved in nitric oxide-induced stomatal closure. Planta 236, 1899 1907. doi: 10.1007/s00425-0121745-4

Dutilleul, C., Benhassaine-Kesri, G., Demandre, C., Rézé, N., Launay, A., Pelletier, S., et al. (2012). Phytosphingosine-phosphate is a signal for AtMPK6 activation and Arabidopsis response to chilling. New Phytol. 194, 181-191. doi: 10.1111/j.1469-8137.2011.04017.x

Falcone, S., Perrotta, C., De Palma, C., Pisconti, A., Sciorati, C., Capobianco, A., et al. (2004). Activation of acid sphingomyelinase and its inhibition by the nitric oxide/cyclic guanosine $3^{\prime}, 5^{\prime}$-monophosphate pathway: key events in Escherichia coli-elicited apoptosis of dendritic cells. J. Immunol. 173, 4452-4463.

Fernández-Marcos, M., Sanz, L., Lewis, D. R., Muday, G. K., and Lorenzo, O. (2011). Nitric oxide causes root apical meristem defects and growth inhibition while reducing PIN-FORMED 1 (PIN1)-dependent acropetal auxin transport. Proc. Natl. Acad. Sci. U.S.A. 108, 18506-18511. doi: 10.1073/pnas.1108644108

Filippov, A., Orädd, G., and Lindblom, G. (2006). Sphingomyelin structure influences the lateral diffusion and raft formation in lipid bilayers. Biophys. J. 90, 2086-2092. doi: 10.1529/biophysj.105.075150

Franzen, R., Fabbro, D., Aschrafi, A., Pfeilschifter, J., and Huwiler, A. (2002). Nitric oxide induces degradation of the neutral ceramidase in rat renal mesangial cells and is counterregulated by protein kinase C. J.
Biol. Chem. 277, 46184-46190. doi: 10.1074/jbc.M204034200

Gaupels, F., Kuruthukulangarakoola, G. T., and Durner, J. (2011). Upstream and downstream signals of nitric oxide in pathogen defence. Curr. Opin. Plant Biol. 14, 707-714. doi: 10.1016/j.pbi.2011.07.005

Gechev, T. S., Gadjev, I. Z., and Hille, J. (2004). An extensive microarray analysis of AAL-toxin-induced cell death in Arabidopsis thaliana brings new insights into the complexity of programmed cell death in plants. Cell. Mol. Life Sci. 61, 1185-1197. doi: 10.1007/s00018-004-4067-2

Guillas, I., Guellim, A., Rezé, N., and Baudouin, E. (2013). Long chain base changes triggered by a short exposure of Arabidopsis to low temperature are altered by AHb1 non-symbiotic haemoglobin overexpression. Plant Physiol. Biochem. 63, 191-195. doi: 10.1016/j.plaphy.2012.11.020

Guo, L., Mishra, G., Markham, J. E., Li, M., Tawfall, A., Welti, R., et al. (2012). Connections between sphingosine kinase and phospholipase D in the abscisic acid signaling pathway in Arabidopsis. J. Biol. Chem. 287, 82868296. doi: 10.1074/jbc.M111.274274

Guo, L., Mishra, G., Taylor, K., and Wang, X. (2011). Phosphatidic acid binds and stimulates Arabidopsis sphingosine kinases. J. Biol. Chem. 286, 13336-13345. doi: 10.1074/jbc.M110.190892

Guo, L., and Wang, X. (2012). Crosstalk between phospholipase D and sphingosine kinase in plant stress signaling. Front. Plant Sci. 3:51. doi: 10.3389/fpls.2012.00051

Huwiler, A., and Pfeilschifter, J. (2003). Nitric oxide signalling with a special focus on lipid-derived mediators. 
Biol. Chem. 384, 1379-1389. doi: 10.1515/BC.2003.154

Huwiler, A., Pfeilschifter, J., and van den Bosch, H. (1999). Nitric oxide donors induce stress signaling via ceramide formation in rat renal mesangial cells. J. Biol. Chem. 274, 7190-7195. doi: 10.1074/jbc.274.11.7190

Igarashi, J., and Michel, T. (2008). S1P and eNOS regulation. Biochim. Biophys. Acta 1781, 489-495. doi: 10.1016/j.bbalip.2008.06.008

Jacques, S., Ghesquière, B., Van Breusegem, F., and Gevaert, K (2013). Plant proteins under oxidative attack. Proteomics 13, 932-940. doi: 10.1002/pmic.201200237

Jeandroz, S., Lamotte, O., Astier, J., Rasul, S., Trapet, P., Besson-Bard, A., et al. (2013). There's more to the picture than meets the eye: nitric oxide cross-talk with $\mathrm{Ca}^{2+}$ signaling. Plant Physiol. doi: 10.1104/pp.113.220624 [Epub ahead of print].

Kihara, A., Mitsutake, S., Mizutani, Y., and Igarashi, Y. (2007). Metabolism and biological functions of two phosphorylated sphingolipids, sphingosine 1-phosphate, and ceramide 1-phosphate. Prog. Lipid Res. 46, 126-144. doi: 10.1016/j.plipres.2007.03.001

Kohr, M. J., Aponte, A. M., Sun, J., Wang, G., Murphy, E., Gucek, M., et al. (2011). Characterization of potential $S$-nitrosylation sites in the myocardium. Am. J. Physiol. Heart Circ. Physiol. 300, 1327-1335. doi: 10.1152/ajpheart.00997.2010

Kovacs, I., and Lindermayr, C. (2013). Nitric oxide-based protein modification: formation and sitespecificity of protein $S$-nitrosylation. Front. Plant Sci. 4:137. doi: 10.3389/fpls.2013.00137

Lachaud, C., Da Silva, D., Cotelle, V., Thuleau, P., Xiong, T. C., Jauneau, A., etal. (2010). Nuclear calcium controls the apoptotic-like cell death induced by d-erythro-sphinganine in tobacco cells. Cell Calcium 47, 92-100. doi: 10.1016/j.ceca.2009.11. 011

Laxalt, A. M., Raho, N., Have, A. T., and Lamattina, L. (2007). Nitric oxide is critical for inducing phosphatidic acid accumulation in xylanase-elicited tomato cells. J. Biol. Chem. 282, 21160-21168. doi: 10.1074/jbc.M701212200

Lefebvre, B., Furt, F., Hartmann, M.-A., Michaelson, L. V., Carde, J.-P., Sargueil-Boiron, F., et al. (2007). Characterization of lipid rafts from Medicago truncatula root plasma membranes: a proteomic study reveals the presence of a raft-associated redox system.
Plant Physiol. 144, 402-418. doi: 10.1104/pp.106.094102

Levine, Y. C., Li, G. K., and Michel, T. (2007). Agonist-modulated regulation of AMP-activated protein kinase (AMPK) in endothelial cells. Evidence for an $\mathrm{AMPK} \rightarrow$ $\mathrm{Racl} \rightarrow$ Akt $\rightarrow$ endothelial nitricoxide synthase pathway. J. Biol. Chem. 282, 20351-20364. doi: 10.1074/jbc.M702182200

Li, X., Wang, X., Yang, Y., Li R., He, Q., Fang, X., et al. (2011). Single-molecule analysis of PIP2;1 dynamics and partitioning reveals multiple modes of Arabidopsis plasma membrane aquaporin regulation. Plant Cell 23, 3780-3797. doi: 10.1105/tpc.111.091454

Liang, H., Yao, N., Song, L. T., Luo, S., Lu, H., and Greenberg, L. T. (2003). Ceramides modulate programmed cell death in plants. Genes Dev. 17, 2636-2641. doi: 10.1101/gad.1140503

Lombardo, M. C., and Lamattina, L. (2012). Nitric oxide is essential for vesicle formation and trafficking in Arabidopsis root hair growth. J. Exp. Bot. 63, 4875-4885. doi: 10.1093/jxb/ers 166

Markham, J. E., Lynch, D. V., Napier, J. A., Dunn, T. M., and Cahoon, E. B. (2013). Plant sphingolipids: function follows form. Curr. Opin. Plant Biol. 16, 350-357. doi: 10.1016/j.pbi.2013.02.009

Markham, J. E., Molino, D., Gissot, L., Bellec, Y., Hématy, K., Marion, J., et al. (2011). Sphingolipids containing very-long-chain fatty acids define a secretory pathway for specific polar plasma membrane protein targeting in Arabidopsis. Plant Cell 23, 23622378. doi: 10.1105/tpc.110.080473

Martínez-Ruiz, A., Cadenas, S., and Lamas, S. (2011). Nitric oxide signaling: classical, less classical, and non-classical mechanisms. Free Radic. Biol. Med. 51, 17-29. doi: 10.1016/j.freeradbiomed.2011.04.010 Minami, A., Fujiwara, M., Furuto, A., Fukao, Y., Yamashita, T., Kamo, M., et al. (2009). Alterations in detergent-resistant plasma membrane microdomains in Arabidopsis thaliana during cold acclimation. Plant Cell Physiol. 50, 341-359. doi: 10.1093/pcp/pcn202

Minami, A., Furuto, A., and Uemura, M. (2010). Dynamic compositional changes of detergent-resistant plasma membrane microdomains during plant cold acclimation. Plant Signal. Behav. 5, 1115-1118. doi: 10.4161/psb.5.9.12478

Morel, J., Claverol, S., Mongrand, S., Furt, F., Fromentin, J., Bessoule, J.-J., etal. (2006). Proteomics of plant detergent-resistant membranes. $\mathrm{Mol}$ Cell. Proteomics 5, 1396-1411. doi: 10.1074/mcp.M600044-MCP200

Mur, L. A. J., Mandon, J., Persijn, S. Cristescu, S. M., Moshkov, I. E., Novikova, G. V., et al. (2013). Nitric oxide in plants: an assessment of the current state of knowledge. $A o B$ Plants 5, pls052. doi: 10.1093/aob$\mathrm{pla} / \mathrm{pls} 052$

Ng, C. K. Y., Carr, K., McAinsh, M. R., Powell, B., and Hetherington, A. M. (2001). Drought-induced guard cell signal transduction involves sphingosine-1-phosphate. Nature 410, 596-599. doi: 10.1038/35069092

Pata, M. O., Hannun, Y. A., and Ng, C. K.-Y. (2010). Plant sphingolipids: decoding the enigma of the Sphinx. New Phytol. 185, 611-630. doi 10.1111/j.1469-8137.2009.03123.x

Peer, M., Bach, M., Mueller, M. J., and Waller, F. (2011). Free sphingobases induce RBOHDdependent reactive oxygen species production in Arabidopsis leaves. FEBS Lett. 585, 3006-3010. doi: 10.1016/j.febslet.2011.08.016

Peer, M., Stegmann, M., Mueller, M. J., and Waller, F. (2010). Pseudomonas syringae infection triggers de novo synthesis of phytosphingosine from sphinganine in Arabidopsis thaliana. FEBS Lett. 584, 4053-4056. doi: 10.1016/j.febslet.2010.08.027

Perrotta, C., Bizzozero, L., Falcone, S., Rovere-Querini, P., Prinetti, A., Schuchman, E. H., et al. (2007). Nitric oxide boosts chemoimmunotherapy via inhibition of acid sphingomyelinase in a mouse model of melanoma. Cancer Res. 67, 7559-7564. doi: 10.1158/0008-5472.CAN-07-0309

Perrotta, C., and Clementi, E. (2010). Biological roles of acid and neutral sphingomyelinases and their regulation by nitric oxide. Physiology 25, 64-71. doi: 10.1152/physiol.00048.2009

Perrotta, C., De Palma, C., and Clementi, E. (2008). Nitric oxide and sphingolipids: mechanisms of interaction and role in cellular pathophysiology. Biol. Chem. 389, 1391-1397. doi: 10.1515/BC.2008.155

Pilane, C. M., and LaBelle, E. F. (2004). NO induced apoptosis of vascular smooth muscle cells accompanied by ceramide increase. J. Cell. Physiol. 199, 310-315. doi: 10.1002/jcp.10464 Roudier, F., Gissot, L., Beaudoin, F., Haslam, R., Michaelson, L., Marion, J., et al. (2010). Very-long-chain fatty acids are involved in polar auxin transport and developmental patterning in
Arabidopsis. Plant Cell 22, 364-375. doi: 10.1105/tpc.109.071209

Saucedo-Garcia, M., Guevara-Garcia, A., Gonzalez-Solis, A., Cruz-Garcia, F., Vazquez-Santana, S., Markham, J. E., et al. (2011). MPK6, sphinganine and the LCB2a gene from serine palmitoyltransferase are required in the signaling pathway that mediates cell death induced by long chain bases in Arabidopsis. New Phytol. 191, 943-957. doi: 10.1111/j.14698137.2011.03727.x

Schwalm, S., Pfeilschifter, J., and Huwiler, A. (2010). Sphingosine kinase 1 is critically involved in nitric oxide-mediated human endothelial cell migration and tube formation. Br. J. Pharmacol. 160, 1641-1651. doi: 10.1111/j.1476-5381.2010.00818.x

Shi, L. H., Bielawski, J., Mu, J. Y., Dong, H. L., Teng, C., Zhang, J., et al. (2007). Involvement of sphingoid bases in mediating reactive oxygen intermediate production and programmed cell death in Arabidopsis. Cell Res. 17, 1030-1040. doi: 10.1038/cr.2007. 100

Simon-Plas, F., Perraki, A., Bayer, E., Gerbeau-Pissot, P., and Mongrand, S. (2011). An update on plant membrane rafts. Curr. Opin. Plant Biol. 14, 642-649. doi: 10.1016/j.pbi.2011.08.003

Takeda, Y., Tashima, M., Takahashi, A., Uchiyama, T., and Okazaki, T. (1999). Ceramide generation in nitric oxideinduced apoptosis. Activation of magnesium-dependent neutral sphingomyelinase via caspase-3. J. Biol. Chem. 274, 10654-10660. doi: 10.1074/jbc.274.15.10654

Ternes, P., Feussner, K., Werner, S., Lerche, J., Iven, T., Heilmann, I., et al. (2011). Disruption of the ceramide synthase LOH1 causes spontaneous cell death in Arabidopsis thaliana. New Phytol. 192, 841-854. doi: 10.1111/j.1469-8137.2011.03852.x

Umemura, K., Tanino, S., Nagatsuka, T., Koga, J., Iwata, M., Nagashima, K., et al. (2004). Cerebroside elicitor confers resistance to fusarium disease in various plant species. Phytopathology 94, 813-818. doi: 10.1094/PHYTO.2004.94.8.813

Uraji, M., Katagiri, T., Okuma, E., Ye, W., Hossain, M. A., Masuda, C., et al. (2012). Cooperative function of PLD $\delta$ and PLD $\alpha 1$ in abscisic acid-induced stomatal closure in Arabidopsis. Plant Physiol. 159, 450-460. doi: 10.1104/pp.112.195578

Wang, J., Zheng, L., and Tan, R. (2007). Involvement of nitric oxide in cerebroside-induced defense responses and taxol production in Taxus yunnanensis suspension cells. 
Appl. Microbiol. Biotechnol. 75, 1183 1190. doi: 10.1007/s00253-0070927-7

Wang, J. W., Zheng, L. P., Zhang, B., and Zou, T. (2009). Stimulation of artemisinin synthesis by combined cerebroside and nitric oxide elicitation in Artemisia annua hairy roots. Appl. Microbiol. Biotechnol. 85, 285292. doi: 10.1007/s00253-009-2090-9

Wang, W. M., Yang, X. H., Tangchaiburana, S., Ndeh, R., Markham, J. E., Tsegaye, Y., et al. (2008). An inositolphosphorylceramide synthase is involved in regulation of plant programmed cell death associated with defense in Arabidopsis. Plant Cell 20, 3163-3179. doi: 10.1105/tpc.108.060053

Worrall, D., Liang, Y. K., Alvarez, S., Holroyd, G. H., Spiegel, S., Panagopulos, M., et al. (2008). Involvement of sphingosine kinase in plant cell signalling. Plant J. 56, 64-72. doi: 10.1111/j.1365-313X.2008.03579.x

Yang, H., Richter, G. L., Wang, X., Młodzińska, E., Carraro, N., Ma, G., etal. (2013). Sterols and sphingolipids differentially function in trafficking of the Arabidopsis ABCB19 auxin transporter. Plant J. 74, 37-47. doi: $10.1111 /$ tpj.12103

Zhan, X., and Desiderio, D. M (2009). Mass spectrometric identification of in vivo nitrotyrosine sites in the human pituitary tumor proteome. Methods Mol. Biol. 566, 137 163. doi: 10.1007/978-1-59745-5626_10

Zhang, H., Zhai, J., Mo, J., Li, D. and Song, F. (2012). Overexpression of rice sphingosine-1-phosphate lyase gene OsSPL1 in transgenic tobacco reduces salt and oxidative stress tolerance. J. Integr. Plant Biol. 54, 652-662. doi: 10.1111/j.17447909.2012.01150.x

Zhang, Y., Zhu, H., Zhang, Q., Li, M., Yan, M., Wang, R., etal. (2009). Phospholipase Dalphal and phosphatidic acid regulate NADPH oxidase activity and production of reactive oxygen species in ABAmediated stomatal closure in Arabidopsis. Plant Cell 21, 2357-2377. doi: $10.1105 /$ tpc.108.062992

Conflict of Interest Statement: The authors declare that the research was conducted in the absence of any commercial or financial relationships that could be construed as a potential conflict of interest.

Received: 04 July 2013; paper pending published: 17 July 2013; accepted:
13 August 2013; published online: 12 September 2013.

Citation: Guillas I, Puyaubert J and Baudouin E (2013) Nitric oxide-sphingolipid interplays in plant signaling: a new enigma from the Sphinx? Front. Plant Sci. 4:341. doi: 10.3389/fpls.2013.00341

This article was submitted to Plant Physiology, a section of the journal Frontiers in Plant Science.

Copyright (c) 2013 Guillas, Puyaubert and Baudouin. This is an open-access article distributed under the terms of the Creative Commons Attribution License (CC BY). The use, distribution or reproduction in other forums is permitted, provided the original author(s) or licensor are credited and that the original publication in this journal is cited, in accordance with accepted academic practice. No use, distribution or reproduction is permitted which does not comply with these terms. 